\title{
ORDER EMBEDDING OF A MATRIX ORDERED SPACE
}

\author{
ANIL K. KARN
}

(Received 16 January 2010)

\begin{abstract}
We characterize certain properties in a matrix ordered space in order to embed it in a $C^{*}$-algebra. Let such spaces be called $C^{*}$-ordered operator spaces. We show that for every self-adjoint operator space there exists a matrix order (on it) to make it a $C^{*}$-ordered operator space. However, the operator space dual of a (nontrivial) $C^{*}$-ordered operator space cannot be embedded in any $C^{*}$-algebra.

2010 Mathematics subject classification: primary 46L07.

Keywords and phrases: matrix ordered space, operator space, operator system, $L^{\infty}$-matricially Riesz normed space, $C^{*}$-ordered operator space, $C^{*}$-matricially Riesz normed space.
\end{abstract}

\section{The characterization theorem}

In this short communication, we determine a set of necessary and sufficient conditions on a matrix ordered space so that it can be order embedded in some $C^{*}$-algebra. (Some related results can be found in $[6,12]$.) Let us call such spaces $C^{*}$-ordered operator spaces. We have been able to show that on any self-adjoint operator space there exists a matrix order (which may be trivial) such that the space turns out to be a $C^{*}$-ordered operator space. Interestingly, however, we have proved that the operator space dual of a (nontrivial) $C^{*}$-ordered operator space is not a $C^{*}$-ordered operator space. In particular, the operator space dual of an operator system cannot be order embedded in a $C^{*}$-algebra. This improves a result due to Blecher and Neal [1]. At the end of this paper, we discuss a class of examples of $C^{*}$-ordered operator spaces.

We begin by recalling some definitions. Let $V$ be a complex vector space. For $m, n \in \mathbf{N}, M_{m, n}(V)$ denotes the set of all $m \times n$ matrices with entries from $V$. For $m=n$, we write, $M_{m, n}(V)=M_{n}(V)$. When $V=\mathbf{C}$, we write $M_{m, n}(V)=M_{m, n}$.

DEFINITION 1.1. An $L^{\infty}$-matricially normed space (that is, an abstract operator space [10]), denoted by $\left(V,\left\{\|\|_{n}\right\}\right)$, is a complex vector space $V$ together with a sequence of norms \|\|$_{n}$ (called a matrix norm on $V$ ) such that:

(i) $\left(M_{n}(V),\|\|_{n}\right)$ is a normed linear space for all $n$;

(ii) $\|v \oplus w\|_{n+m}=\max \left\{\|v\|_{n},\|w\|_{m}\right\}$; and

(iii) $\|\alpha v \beta\|_{n} \leq\|\alpha\|\|v\|_{n}\|\beta\|$ for all $v \in M_{n}(V), w \in M_{m}(V), \alpha, \beta \in M_{n}$ and $n \in \mathbf{N}$.

(C) 2011 Australian Mathematical Publishing Association Inc. 0004-9727/2011 \$16.00 
Definition 1.2. A *-vector space is complex vector space $V$ together with an involution $*$. A matrix ordered space is a $*$-vector space $V$ together with a cone $M_{n}(V)^{+}$in $M_{n}(V)_{\text {sa }}$ for all $n \in \mathbf{N}$ and with the following property: if $v \in M_{n}(V)^{+}$and $\gamma \in M_{n, m}$ then $\gamma^{*} v \gamma \in M_{m}(V)^{+}$for any $n, m \in \mathbf{N}$. It is denoted by $\left(V,\left\{M_{n}(V)^{+}\right\}\right)$.

DEFINITION 1.3. An $L^{\infty}$-matricially $*$-normed space is an $L^{\infty}$-matricially normed space $\left(V,\left\{\|\|_{n}\right\}\right)$, such that $V$ is a $*$-vector space and that for all $v \in M_{n}(V)$ we have $\left\|v^{*}\right\|_{n}=\|v\|_{n}$.

DEFINITION 1.4. Let $V$ and $W$ be complex vector spaces. Every linear map $\phi$ : $V \rightarrow W$ induces a sequence $\left\{\phi_{n}\right\}$ where $\phi_{n}\left(\left[v_{i j}\right]\right)=\left[\phi\left(v_{i j}\right)\right]$. Let $\left(V,\left\{\|\|_{n}\right\}\right)$ and $\left(W,\left\{\|\|_{n}\right\}\right)$ be $L^{\infty}$-matricially normed spaces. Then a linear map $\phi: V \rightarrow W$ is called completely bounded if $\|\phi\|_{\mathrm{cb}}=\sup \left\{\left\|\phi_{n}\right\|: n \in \mathbf{N}\right\}<\infty$ and $\phi$ is called a complete isometry if $\phi_{n}$ is an isometry for all $n$. Let $\left(V,\left\{M_{n}(V)^{+}\right\}\right)$and $\left(W,\left\{M_{n}(W)^{+}\right\}\right)$be matrix ordered spaces and let $\phi: V \rightarrow W$ be a self-adjoint linear map. We say that $\phi$ is completely positive if $\phi_{n}$ is positive for all $n$, and that $\phi$ is a complete order isomorphism if it is a linear isomorphism and both $\phi$ and $\phi^{-1}$ are completely positive on their domains.

Definition 1.5. Let $\left(V,\left\{M_{n}(V)^{+}\right\}\right)$be a matrix ordered space. We say that $V^{+}$is proper if $V^{+} \cap\left(-V^{+}\right)=\{0\}$.

It is shown in [2] that if $V^{+}$is proper, then so is $M_{n}(V)^{+}$for all $n$. In the first result we extract some necessary conditions on a matrix ordered space so that it may be embedded in a $C^{*}$-algebra. We also prove that these conditions are sufficient.

Proposition 1.6. Let $\left(V,\left\{M_{n}(V)^{+}\right\}\right)$be a matrix ordered space. Assume that $\phi: V \rightarrow A$ is a linear complete order isomorphism for some $C^{*}$-algebra A. For each $n \in \mathbf{N}$ define

$$
\|v\|_{n}=\left\|\phi_{n}(v)\right\|
$$

for all $v \in M_{n}(V)$. Then:

(1) $\left(V,\left\{\|\|_{n}\right\}\right)$ is an (abstract) operator space;

(2) $\left\|v^{*}\right\|_{n}=\|v\|_{n}$ for all $v \in M_{n}(V), n \in \mathbf{N}$. (In other words, $V$ is an $L^{\infty}$ matricially $*$-normed space.)

Put $Q_{n}(V)=\left\{f: M_{n}(V) \rightarrow \mathbf{C} \mid f \geq 0\right.$ and $\left.\|f\| \leq 1\right\}$ for all $n \in \mathbf{N}$. Then:

(3) $\|v\|=\sup \left\{|f(v)|: f \in Q_{n}(V)\right\}$ for all $v \in M_{n}(V)_{\mathrm{sa}}, n \in \mathbf{N}$;

(4) for $n \in \mathbf{N}$ and $v \in M_{n}(V)_{\mathrm{sa}}$ we have $v \in M_{n}(V)^{+}$if and only if $f(v) \geq 0$ for all $f \in Q_{n}(V)$;

(5) $V^{+}$(and therefore $M_{n}(V)^{+}$, for all $n$ ) is proper.

PROOF. (1) By definition, $\phi$ becomes a complete isometry so that $V$ may be treated as a subspace of $A$.

(2) For any $v \in M_{n}(V)$,

$$
\left\|v^{*}\right\|_{n}=\left\|\phi_{n}\left(v^{*}\right)\right\|=\left\|\phi_{n}(v)^{*}\right\|=\left\|\phi_{n}(v)\right\|=\|v\|_{n} .
$$


(3) We know that for all $a \in M_{n}(A)_{\text {sa }}$,

$$
\|a\|=\sup \left\{|g(a)|: g \in Q_{n}(A)\right\} .
$$

Also, for $g \in Q_{n}(A)$, we have $g \circ \phi_{n} \in Q_{n}(V)$. Thus

$$
\|v\|_{n}=\left\|\phi_{n}(v)\right\|=\sup \left\{\left|g\left(\phi_{n}(v)\right)\right|: g \in Q_{n}(A)\right\} \leq \sup \left\{|f(v)|: f \in Q_{n}(V)\right\} .
$$

The other part is obvious.

(4) First, let $v \in M_{n}(V)_{\text {sa }}$ be such that $f(v) \geq 0$ for all $f \in Q_{n}(V)$. Then as in (3) we have that $g \circ \phi_{n}(v) \geq 0$ for all $g \in Q_{n}(A)$. It follows that $\phi_{n}(v) \in M_{n}(A)^{+}$. Since $\phi$ is a complete order isomorphism, we may conclude that $v \in M_{n}(V)^{+}$. Now the converse is trivial. Finally, as $\phi$ is a (complete) order isomorphism, (5) also holds.

We shall also prove the converse of this result. For this purpose, we shall require the following improvement on a result due to Effros and Ruan [3].

THEOREM 1.7. Let $\left(V,\left\{M_{n}(V)^{+}\right\}\right)$be a matrix ordered space. Assume that $\left\{\|\|_{n}\right\}$ is a matrix norm on $V$ such that it is an $L^{\infty}$-matricially $*$-normed space. Fix $n \in \mathbf{N}$ and let $f: M_{n}(V) \rightarrow \mathbf{C}$ be a linear self-adjoint contraction. Then there exist a linear, self-adjoint, complete contraction $\phi: V \rightarrow M_{n}$ and a norm-one $n^{2} \times 1$ matrix $\delta$ such that

$$
f(v)=\delta^{*} \phi_{n} \delta
$$

If in addition, $f$ is positive, then $\phi$ is completely positive too.

PROOF. The techniques used in the proof are essentially adapted from [3]. However, for completeness, we include the main points of the proof. It is divided into several steps.

Step I. Consider the $C^{*}$-algebra $M_{n}$ and let $S$ be its state space. Let $C(S)$ denote the space of all real-valued, continuous functions on $S$. For $\alpha \in M_{m, n}$ and $v \in M_{m}(V)_{\text {sa }}$ with $\|v\|_{m}=1$, we define $\psi_{v}^{\alpha} \in C(S)$ given by

$$
\psi_{v}^{\alpha}(p)=p\left(\alpha^{*} \alpha\right)-f\left(\alpha^{*} v \alpha\right)
$$

for all $p \in S$. Put

$$
\Psi=\left\{\psi_{v}^{\alpha}: \alpha \in M_{m, n} \text { and } v \in M_{m}(V)_{\mathrm{sa}} \text { with }\|v\|_{m}=1\right\} .
$$

Then $\psi_{v}^{\alpha}+\psi_{w}^{\beta}=\psi_{v \oplus w}^{\left[\begin{array}{c}\alpha \\ \beta\end{array}\right]}$ and $|\lambda|^{2} \psi_{v}^{\alpha}=\psi_{v}^{\lambda \alpha} \quad$ for all $\alpha \in M_{m, n}, \beta \in M_{p, n}, v \in$ $M_{m}(V)_{\mathrm{sa}}, w \in M_{p}(V)_{\text {sa }}$ with $\|v\|_{m}=1,\|w\|_{p}=1$ and $\lambda \in \mathbf{C}$. Since $\left\{\|\|_{n}\right\}$ satisfies the $L^{\infty}$-condition, we see that $\|v \oplus w\|_{m+p}=1$. Thus $\Psi$ is a cone. Let $\Gamma$ denote the cone of all strictly negative functions in $C(S)$. Then $\operatorname{int}(\Gamma) \neq \emptyset$ and $\Psi \cap \Gamma=\emptyset$. Thus by the geometric form of the Hahn-Banach theorem, there exists a nonzero Radon measure $\mu$ on $S$ such that $\mu \mid \Psi \geq 0$ and $\mu \mid \Gamma \leq 0$. It follows that $\mu$ is a positive measure 
and we may assume that it is a probability measure. Then $p_{0}=\int_{S} p d \mu(p) \in S$. Since $\mu \mid \Psi \geq 0$, for any $\alpha \in M_{m, n}$ and $v \in M_{m}(V)_{\text {sa }}$ with $\|v\|_{m}=1$ we have

$$
0 \leq \int_{S} \Psi_{v}^{\alpha}(p) d \mu(p)=\Psi_{v}^{\alpha}\left(p_{0}\right)=p_{0}\left(\alpha^{*} \alpha\right)-f\left(\alpha^{*} v \alpha\right) .
$$

In other words,

$$
f\left(\alpha^{*} v \alpha\right) \leq p\left(\alpha^{*} \alpha\right)\|v\|_{m},
$$

for all $\alpha \in M_{m, n}$ and $v \in M_{m}(V)_{\text {sa }}$. Now using standard techniques (see, for example, [3]), we may conclude that

$$
f\left(\alpha^{*} v \beta\right) \leq\left[p\left(\alpha^{*} \alpha\right) p\left(\beta^{*} \beta\right)\right]^{1 / 2}\|v\|_{m},
$$

for all $\alpha, \beta \in M_{m, n}$ and $v \in M_{m}(V)$.

Step II. Let $\left\{\varepsilon_{i j}: 1 \leq i, j \leq n\right\}$ be the matrix units of $M_{n}$. Put $p_{0}\left(\varepsilon_{i j}\right)=\alpha_{j i}$ and set $A_{0}=\left[\alpha_{i j}\right] \in M_{n}$. It follows, from [5, Exercise 4.6.18], that:

(1) $A_{0} \in M_{n}^{+}$;

(2) $\operatorname{tr}\left(A_{0}\right)=1$; and

(3) $p_{0}(B)=\operatorname{tr}\left(A_{0} B\right)$ for all $B \in M_{n}$.

Let $A$ be the positive square root of $A_{0}$. Consider the closed subspace $K=A\left(\mathbf{C}^{n}\right)$ of $\mathbf{C}^{n}$. For a fixed $v \in V$, define $\hat{v}: K \times K \rightarrow \mathbf{C}$ given by

$$
\hat{v}(A(\alpha), A(\beta))=f\left(\alpha v \beta^{*}\right)
$$

for all $\alpha, \beta \in \mathbf{C}^{n}$ (identified with $M_{n, 1}$ ). Then $\hat{v}$ is a contractive sesquilinear form on $K$, for $\|A(\alpha)\|^{2}=p_{0}\left(\alpha \alpha^{*}\right)$ by (3). Thus there exists a unique contractive linear map $T_{v}: K \rightarrow K$ such that

$$
\left\langle T_{v} A(\alpha), A(\beta)\right\rangle=f\left(\alpha v \beta^{*}\right) .
$$

Let $P$ be the range projection of $A$. Then $\phi(v)=T_{v} P$ may be identified in $M_{n}$ and we may conclude that $v \mapsto \phi(v)$ defines a self-adjoint linear map $\phi: V \rightarrow M_{n}$. Let $\left\{\varepsilon_{i}: 1 \leq i \leq n\right\}$ be the matrix units of $M_{n, 1}$. Set $\delta=\left(A\left(\varepsilon_{i}\right)\right) \in\left(\mathbf{C}^{n}\right)^{n}$ (identified with $\left.M_{n^{2}, 1}\right)$. Then

$$
\|\delta\|^{2}=\sum_{i=1}^{n}\left\|A\left(\varepsilon_{i}\right)\right\|^{2}=\operatorname{tr}\left(A_{0}\right)=1
$$

using (2). Since for $v=\left[v_{i j}\right] \in M_{n}(V)$ we have $v=\sum_{i, j=1}^{n} \varepsilon_{i} v_{i j} \varepsilon_{j}^{*}$, we obtain that

$$
f(v)=\sum_{i, j=1}^{n}\left\langle\phi\left(v_{i j}\right) A\left(\varepsilon_{i}\right), A\left(\varepsilon_{j}\right)\right\rangle=\delta^{*} \phi_{n}(v) \delta .
$$

Now the rest of the proof is routine.

THEOREM 1.8. Let $\left(V,\left\{M_{n}(V)^{+}\right\}\right)$be a matrix ordered space. Assume that $\left\{\|\|_{n}\right\}$ is a matrix norm on $V$ and that conditions (1)-(5) of Proposition 1.6 hold in $V$. Then there exist a $C^{*}$-algebra $A$ and a linear, completely isometric, complete order isomorphism $\Phi: V \rightarrow A$. 
Proof. Let us write $C Q_{n}(V)$ for the set of all completely contractive completely positive maps $\phi: V \rightarrow M_{n}$. Then $C Q_{n}(V)$ is nonempty. Write $M_{n}^{\phi}$ for $M_{n}$ for all $\phi \in C Q_{n}(V)$ and put $A_{n}=\bigoplus M_{n}^{\phi}$ where $\phi$ runs over $C Q_{n}(V)$ for all $n$. Define $\Phi^{(n)}: V \rightarrow A_{2 n}$ given by

$$
\Phi^{(n)}(v)=(\phi(v))_{\phi \in C Q_{2 n}(V)} .
$$

Then $\Phi^{(n)}$ is a well-defined completely contractive completely positive map. We show that $\left(\Phi^{(n)}\right)_{n}$ is an order isomorphism (onto its range). Let $v \in M_{n}(V)_{\text {sa }}$ be such that $\left(\Phi^{(n)}\right)_{n}(v) \geq 0$. Then $\phi_{n}(v) \in M_{n}\left(M_{2 n}\right)^{+}$for all $\phi \in C Q_{2 n}(V)$. Let $f \in Q_{n}(V)$. Then by Theorem 1.7, there exist $\phi \in C Q_{n}(V)\left(\subset C Q_{2 n}(V)\right)$ and $\delta \in M_{n^{2}, 1}$ such that

$$
f(v)=\delta^{*} \phi_{n}(v) \delta \geq 0 .
$$

Thus by condition (4), $v \in M_{n}(V)^{+}$. Next, let $v \in M_{n}(V)_{\text {sa }}$ be such that $\left(\Phi^{(n)}\right)_{n}(v)=0$. Then as above, $\pm v \in M_{n}(V)^{+}$so that by condition (5), $v=0$. Thus $\left(\Phi^{(n)}\right)_{n}$ is an order isomorphism for all $n$. Now set

$$
A=\bigoplus\left\{A_{2 n}: n \in \mathbf{N}\right\} \quad \text { (the } C^{*} \text {-direct sum). }
$$

Define $\Phi: V \rightarrow A$ given by $\Phi(v)=\left(\Phi^{(n)}(v)\right)$, for all $v \in V$. Then $\Phi$ is a linear completely contractive complete order isomorphism. We further show that $\Phi$ is a complete isometry. Let $v \in M_{n}(V)$. Then

$$
\left[\begin{array}{cc}
0 & v \\
v^{*} & 0
\end{array}\right] \in M_{2 n}(V)_{\mathrm{sa}}
$$

Thus by condition (4) for given $\epsilon>0$, there is an $f \in Q_{2 n}$ such that

$$
\|v\|_{n}-\epsilon=\left\|\left[\begin{array}{cc}
0 & v \\
v^{*} & 0
\end{array}\right]\right\|_{2 n}-\epsilon<\left\langle f,\left[\begin{array}{cc}
0 & v \\
v^{*} & 0
\end{array}\right]\right\rangle .
$$

By Theorem 1.7 there exist $\phi \in C Q_{2 n}(V)$ and $\delta \in M_{(2 n)^{2}, 1}$ such that

$$
\left|\left\langle f,\left[\begin{array}{cc}
0 & v \\
v^{*} & 0
\end{array}\right]\right\rangle\right|=\delta^{*} \phi_{2 n}\left(\left[\begin{array}{cc}
0 & v \\
v^{*} & 0
\end{array}\right]\right) \delta .
$$

Since

$$
\left|\delta^{*} \phi_{2 n}\left(\left[\begin{array}{cc}
0 & v \\
v^{*} & 0
\end{array}\right]\right) \delta\right| \leq\left\|\phi_{2 n}\left(\left[\begin{array}{cc}
0 & v \\
v^{*} & 0
\end{array}\right]\right)\right\|=\left\|\phi_{n}(v)\right\| \leq\|v\|_{n}
$$

and since $\epsilon>0$ is arbitrary, we conclude that $(\phi)_{n}$ is an isometry for all $n$. Now the result is immediate.

Definition 1.9. An operator space considered in Theorem 1.8 will be called a $C^{*}$-ordered operator space. 
Now we shall take another approach to examine $C^{*}$-ordered operator spaces. To begin with, we state the following improvement on a characterization theorem due to Effros and Ruan [3]. A proof may be extracted from the proofs of Theorems 1.7 and 1.8 .

PROPOSITION 1.10. Let $V$ be an $L^{\infty}$-matricially $*$-normed space. Then there exist a $C^{*}$-algebra $A$ and a completely isometric, self-adjoint, linear isomorphism $\phi: V \rightarrow A$.

THEOREM 1.11. Let $V$ be an $L^{\infty}$-matricially $*$-normed space. Then there exists $a$ matrix order structure $\left\{M_{n}(V)^{+}\right\}$on it so that it is a $C^{*}$-ordered operator space.

ProOf. By Proposition 1.10, there exist a $C^{*}$-algebra $A$ and a completely isometric, self-adjoint, linear isomorphism $\phi: V \rightarrow A$. For each natural number $n$, set

$$
M_{n}(V)^{+}=\left\{v \in M_{n}(V)_{\mathrm{sa}}: f \circ \phi_{n}(v) \geq 0 \text { for all } f \in Q_{n}(A)\right\} .
$$

It is routine to check that $\left\{M_{n}(V)^{+}\right\}$is a matrix order on $V$ and that $V^{+}$is proper. Moreover, since $A$ is a $C^{*}$-algebra, we also have

$$
\|v\|_{n}=\left\|\phi_{n}(v)\right\|=\sup \left\{\left|f \circ \phi_{n}(v)\right|: f \in Q_{n}(A)\right\} .
$$

Now, by construction, $f \circ \phi_{n} \in Q_{n}(V)$ for all $f \in Q_{n}(A)$ so that $\phi$ has the required properties to complete the proof.

\section{Order embedding and operator space duality}

In this section we show that, in general, operator space duality is not suitable for $C^{*}$ ordered operator spaces. At the end, we describe a class of examples of $C^{*}$-operator spaces.

Proposition 2.1. Let $V$ be a $C^{*}$-ordered operator space. Then for any $n \in \mathbf{N}$ and $u, v, w \in M_{n}(V)_{\mathrm{sa}}$, with $u \leq v \leq w$,

$$
\|v\|_{n} \leq \max \left\{\|u\|_{n},\|w\|_{n}\right\} .
$$

In particular, given $n \in \mathbf{N}$ and $\left[\begin{array}{ll}u_{1} & v \\ v^{*} & u_{2}\end{array}\right] \in M_{2 n}(V)^{+}$for some $v \in M_{n}(V)$ and $u_{1}, u_{2} \in$ $M_{n}(V)^{+}$we have

$$
\|v\|_{n} \leq \max \left\{\left\|u_{1}\right\|_{n},\left\|u_{2}\right\|_{n}\right\} .
$$

Proof. Let $u, v, w \in M_{n}(V)_{\mathrm{sa}}$, with $u \leq v \leq w$ for some $n \in \mathbf{N}$. Then given $f \in$ $Q_{n}(V)$ we have $f(u) \leq f(v) \leq f(w)$. Thus by the definition, $-\|u\|_{n} \leq|f(v)| \leq$ $\|w\|_{n}$ for all $f \in Q_{n}(V)$. Now it follows that

$$
\|v\|_{n} \leq \max \left\{\|u\|_{n},\|w\|_{n}\right\} .
$$

Next, let $\left[\begin{array}{cc}u_{1} & v \\ v^{*} & u_{2}\end{array}\right] \in M_{2 n}(V)^{+}$for some $v \in M_{n}(V)$ and $u_{1}, u_{2} \in M_{n}(V)^{+}$. Then

$$
-\left[\begin{array}{cc}
u_{1} & 0 \\
0 & u_{2}
\end{array}\right] \leq\left[\begin{array}{cc}
0 & v \\
v^{*} & 0
\end{array}\right] \leq\left[\begin{array}{cc}
u_{1} & 0 \\
0 & u_{2}
\end{array}\right] \in M_{2 n}(V)^{+} .
$$

Now by the first part, the result is immediate, for $V$ is an operator space. 
THEOREM 2.2. Let $V$ be a nonzero $C^{*}$-ordered operator space. If the operator space dual $V^{\prime}$ of $V$ is also a $C^{*}$-ordered operator space, then $V \cong \mathbf{C}$.

PROOF. Let $f$ be a bounded self-adjoint linear functional on $V$. Since $V$ is $C^{*}$ ordered, by Proposition 2.1 above and [4, Theorem 3.6.2], there are bounded positive linear functionals $g_{1}$ and $g_{2}$ on $V$ such that $f=g_{1}-g_{2}$ with $\left\|g_{1}\right\|+\left\|g_{2}\right\| \leq\|f\|$. Then $-g_{2} \leq f \leq g_{1}$. Thus as $V^{\prime}$ is also $C^{*}$-ordered, by Proposition 2.1, we get that $\|f\| \leq \max \left\{\left\|g_{1}\right\|,\left\|g_{2}\right\|\right\}$. Therefore, $\left\|g_{1}\right\|+\left\|g_{2}\right\| \leq \max \left\{\left\|g_{1}\right\|,\left\|g_{2}\right\|\right\}$. It follows that either $g_{1}=0$ or $g_{2}=0$. In other words, $\left(V^{\prime}\right)_{\mathrm{sa}}=\left(V^{\prime}\right)^{+} \cup\left(-\left(V^{\prime}\right)^{+}\right)$. Thus for any $f, g \in\left(V^{\prime}\right)_{\text {sa }}$, either $f \leq g$ or $g \leq f$. Consider

$$
Q(V)=\left\{f \in\left(V^{\prime}\right)^{+}:\|f\| \leq 1\right\} .
$$

Then $Q(V)$ is nonempty, weak*-compact and convex. Let $e_{1}$ and $e_{2}$ be any two nonzero extreme points of $Q(V)$. Then as above, these are comparable in $\left(V^{\prime}\right)_{\mathrm{sa}}$. For definiteness, we may assume that $e_{1} \leq e_{2}$. If $e_{1} \neq e_{2}$, then

$$
e_{2}=\frac{1}{2}\left(e_{2}-e_{1}\right)+\frac{1}{2}\left(e_{2}+e_{1}\right)
$$

is a proper convex combination in $Q(V)$. Since $e_{2}$ is an extreme point of $\mathbf{C}$, we have either $\frac{1}{2}\left(e_{2}-e_{1}\right)=0$ or $\frac{1}{2}\left(e_{2}+e_{1}\right)=0$. Since $e_{1}$ and $e_{2}$ are nonzero, we must have $e_{1}=e_{2}$. In other words, $Q(V)$ has a unique nonzero extreme point, say $e_{0}$. Since 0 is also an extreme point of $Q(V)$, for any $f \in Q(V)$ we get, by the Krien-Milman theorem, that $f=k e_{0}$ for some $k \in[0,1]$. Then $\left\|e_{0}\right\|=1$. Now it is immediate that $V \cong \mathbf{C}$.

The following result due to Blecher and Neal [1] is a special case of the above result.

COROLlary 2.3. The operator space dual of a nonscalar $C^{*}$-algebra cannot be order embedded in any $C^{*}$-algebra.

REMARK 2.4. It will not be hard to show that the operator space dual of an $L^{\infty}$ matricially $*$-normed space is again an $L^{\infty}$-matricially $*$-normed space. Thus the operator space duality seems to have a problem with the relation with the matrix norm and the matrix order. However, we are not in a position to comment on this at this moment.

At the end we record that matrix order unit spaces (operator systems) are $C^{*}$ ordered operator spaces. More generally, every approximate matrix order unit space is a $C^{*}$-ordered operator space. The latter class includes the class of operator systems and that of $C^{*}$-algebras (unital or nonunital). These classes possess a structure richer than that of $C^{*}$-ordered operator spaces. We explain this as follows.

Definition 2.5. We say that $V^{+}$is generating if given $v \in V$ there exist $v_{0}, v_{1}, v_{2}, v_{3} \in V^{+}$such that $v=\sum_{k=0}^{3} i^{k} v_{k}$, where $i^{2}=-1$. 
It is proved in [7, Proposition 1.8] that $V^{+}$is generating if and only if given $v \in V$ there are $u_{1}, u_{2} \in V^{+}$such that $\left[\begin{array}{ll}u_{1} & v \\ v^{*} & u_{2}\end{array}\right] \in M_{2}(V)^{+}$and that in this case $M_{n}(V)^{+}$is generating for all $n$. In this case, we say that $\left(V,\left\{M_{n}(V)^{+}\right\}\right)$is a positively generated matrix ordered space.

DEFINITION 2.6. Let $\left(V,\left\{M_{n}(V)^{+}\right\}\right)$be a positively generated matrix ordered space. A norm \|\| on $V$ will be called a Riesz norm if for all $v \in V$,

$$
\|v\|=\left\{\max \left(\left\|u_{1}\right\|,\left\|u_{2}\right\|\right): u_{1}, u_{2} \in V^{+} \text {and }\left[\begin{array}{cc}
u_{1} & v \\
v^{*} & u_{2}
\end{array}\right] \in M_{2}(V)^{+}\right\} .
$$

DEFINITION 2.7. An $L^{\infty}$-matricially Riesz normed space (matrix regular operator space [11]) is a positively generated matrix ordered space $\left(V,\left\{M_{n}(V)^{+}\right\}\right)$together with a matrix norm $\left\{\|\|_{n}\right\}$ such that \|\|$_{n}$ is a Riesz norm on $M_{n}(V)$ and $M_{n}(V)^{+}$is norm closed for all $n$ and that $\left(V,\left\{\|\|_{n}\right\}\right)$ is an $L^{\infty}$-matricially normed space. It is denoted by $\left(V,\left\{\|\|_{n}\right\},\left\{M_{n}(V)^{+}\right\}\right)$. An $L^{\infty}$-matricially Riesz normed space is called a $C^{*}$-matricially Riesz normed space if it is also a $C^{*}$-ordered operator space.

It follows, from Proposition 1.6 and Theorem 1.8 , that an $L^{\infty}$-matricially Riesz normed space can be order embedded in a $C^{*}$-algebra if and only if it is a $C^{*}$ matricially Riesz normed space. Schreiner [11] proved that the operator space dual of an $L^{\infty}$-matricially Riesz normed space is again an $L^{\infty}$-matricially Riesz normed space. It follows, from Theorem 2.2, that, every $L^{\infty}$-matricially Riesz normed space is not a $C^{*}$-matricially Riesz normed space. However, the spaces we define below are $C^{*}$-ordered operator spaces.

Definition 2.8. Let $\left(V,\left\{M_{n}(V)^{+}\right\}\right)$be a matrix ordered space. An increasing net $\left\{e_{\lambda}\right\}$ in $V^{+}$is called an approximate order unit for $V$ if for each $v \in V$ there is a $k>0$ such that

$$
\left[\begin{array}{cc}
k e_{\lambda} & v \\
v^{*} & k e_{\lambda}
\end{array}\right] \in M_{2}(V)^{+} \text {for some } \lambda \text {. }
$$

In this case $\left\{e_{\lambda}^{n}\right\}$ acts as an approximate order unit for $M_{n}(V)$ for all $n$, where $e_{\lambda}^{n}=e_{\lambda} \oplus \cdots \oplus e_{\lambda}$. Moreover, $\left\{e_{\lambda}\right\}$ determines a matrix Riesz seminorm $\left\{\|\|_{n}\right\}$ on $V$. We call $\left(V,\left\{e_{\lambda}\right\}\right)$ an approximate matrix order unit space if $\left(V,\left\{\|\|_{n}\right\},\left\{M_{n}(V)^{+}\right\}\right)$is an $L^{\infty}$-matricially Riesz normed space.

When $e_{\lambda}=e$ for all $\lambda$ we drop the term 'approximate' in the above notions. For example, $(V, e)$ denotes a matrix order unit space. For details, refer to [9].

Let $V$ be an approximate matrix order unit space. It follows, from Theorem 1.8 and [8, Proposition 1.20], that

$$
M_{n}\left(V^{\prime}\right)_{\mathrm{sa}}=\operatorname{co}\left(Q_{n}(V) \cup\left(-Q_{n}(V)\right)\right)
$$

for all $n$. Thus we may conclude with the following result.

PROPOSITION 2.9. An approximate matrix order unit space is a $C^{*}$-matricially Riesz normed space. 


\section{References}

[1] D. P. Blecher and M. Neal, 'Open partial isometries and positivity in operator spaces', Studia Math. 182 (2007), 227-262.

[2] M. D. Choi and E. G. Effros, 'Injectivity and operator spaces', J. Funct. Anal. 24 (1977), 156-209.

[3] E. G. Effros and Z. J. Ruan, 'On the abstract characterization of operator spaces', Proc. Amer. Math. Soc. 119 (1993), 579-584.

[4] G. J. O. Jameson, Ordered Linear Spaces, Lecture Notes in Mathematics, 141 (Springer, New York, 1970).

[5] R. Kadison and J. Ringrose, Fundamentals of the Theories of Operator Algebras, I (Academic Press, New York, 1983).

[6] A. K. Karn, 'A p-theory of ordered normed spaces', Positivity 14 (2010), 441-458.

[7] A. K. Karn and R. Vasudevan, 'Approximate matrix order unit spaces', Yokohama Math. J. 44 (1997), 73-91.

[8] A. K. Karn and R. Vasudevan, 'Matrix duality for matrix ordered spaces', Yokohama Math. J. 45 (1998), 1-18.

[9] A. K. Karn and R. Vasudevan, 'Characterizations of matricially Riesz normed spaces', Yokohama Math. J. 47 (2000), 143-153.

[10] Z. J. Ruan, 'Subspaces of $C^{*}$-algebras', J. Funct. Anal. 76 (1988), 217-230.

[11] W. J. Schreiner, 'Matrix regular operator spaces', J. Funct. Anal. 152 (1998), 136-175.

[12] W. Werner, 'Subspaces of $L(H)$ that are $*$-invariant', J. Funct. Anal. 193 (2002), 207-223.

ANIL K. KARN, Department of Mathematics, Deen Dayal Upadhyaya College, University of Delhi, Karam Pura, New Delhi 110 015, India

e-mail: anil.karn@gmail.com 